\title{
Mathematical Model and Control Simulation of Hexapod Robot Locomotion in Tripod Gait
}

\author{
Krešimir Osman ${ }^{1}$, Trpimir Alajbeg ${ }^{2}$ \\ ${ }^{1}$ Zagreb University of Applied Sciences \\ Department of Electrical Engineering \\ Konavoska 2, 10000 Zagreb, Croatia \\ kresimir.osman@tvz.hr \\ ${ }^{2}$ Zagreb University of Applied Sciences \\ Department of Electrical Engineering \\ Konavoska 2, 10000 Zagreb, Croatia \\ trpimir.alajbeg@tvz.hr
}

\begin{abstract}
This paper presents the research of a hexapod robot as it movement along a defined path. Firstly, the performance of the robot product architecture is presented and a mathematical kinematic model of the robot is derived according to it, i.e. its legs using the Denavit-Hartenberg method and homogeneous transformations. The purpose of this was to obtain the equations of inverse kinematics. A simplified robot product architecture based on rectangular robot design was used to perform the mathematical model. It is shown by a simplified kinematic scheme. In addition, a control algorithm was performed using a PID controller in each ankle. The controller's parameters are adjusted on one leg of the robot, and after that, this is used to adjust the control on the other legs in this way. The simulation of the controlled motion of the robot was performed using the computer program MATLAB Simulink and the Simscape Multibody environment. In doing so, only the robot's movement on flat terrain was observed. The tripod gait was chosen for the motion model due to the simplicity of creating the model and performing the simulation, as well as the speed of movement.
\end{abstract}

Keywords: hexapod robot, tripod gait, rectangular robot design, PID control

\section{INTRODUCTION}

The application of behavioural simulations (kinematic and dynamic model) in robotics have been quite popular in recent years. One of the important reasons is that it allows developers and researchers to predict, test and validate robotic performance before prototyping and production [1]. It also allows faster and easier improvement of management strategies, focused on safety and more cost-effectiveness.

Using knowledge from nature, i.e. by studying and investigating the locomotion of animals helped researchers develop different types of so-called bio-inspired robot [2]. Among these, the most convenient is the researching of arthropods that have six legs and thus simply maintain their stability (insects, centipedes, symphylans, millipedes etc.). They show quite a robustness of the system in case of damage to the legs. One of the typical examples of the development of robots from this group is the hexapod robot based on the anatomy and locomotion of insects.

Hence, researchers encounter the problem of operating such robotic systems, which are quite complex and have an increased number of degrees of freedom (DOF) of motion due to the performance of the legs of robots from multiple ankles (links) [2]. In practice, many commercial six-legged robots have already been carried out, which have found their application in many areas such as [3]: research in remote and inaccessible places (space, seabed, volcanoes, etc.), dangerous environments (like military operations), construction work, transport operations, etc.

Therefore, in hexapod robots, two typical systems at architectures and locomotions originating from spider insects (hexagonal hexapod) and cockroaches (rectangular hexapod) can be found in 
the literature [4]. In this paper, a robot configuration based on a rectangular hexapod system architecture is described. The hexapod robot is a type of mobile robot that achieves its locomotion using six legs (three on each side of chassis), of which at least three or more legs must be on the ground to achieve their stable condition.

When moving in nature, insects can use different types of gait. Inspired by this [5], the most common types of gait they can perform (4 typical walks) of hexapod robots are wave gait, tetrapod gait, transition gait and tripod gait. In this paper, a model of behaviour based on tripod gait is presented. The reason that tripod gait is the most interesting because of movement speed. It is the fastest gait of insects when they maintain their body in dynamic balance [6].

Figure 1 shows the simplified product architecture of a hexapod robot, while figure 2 shows model of one hexapod robot (observed) leg with all the joints and ankles. On this basis, kinematic analysis was done to obtain a mathematical model of the robot. The Denavit-Hartenberg (D-H) method [7]was used to establish the joint coordinate system of a hexapod robot for it's one leg. Individual parameters on each joint and variables on joints (1 - root, 2- hip and 3 - knee joint) are defined, and the equation of motion for the leg is obtained. On each ankle of the open kinematic chain, right-wing orthonormal coordinate systems are systematically joined. As the final equation, a matrix equation is obtained, and the position of the top of the robot's leg relative to the coordinates of the robot's body. Solving the problem of inverse kinematics gives the values of the angles of rotation for each of the joints, i.e., the servo motors on them, for a given point in space at the observed robot leg.

When planning the trajectory of the movement of hexapod robots, the movement of the robot's leg in its stance (supporting) phase and swing (suspending) phase, and the transfer phase should be considered throughout the moving cycle. When performing the behaviour of the robot, the simulation was made for flat terrain. The simulation was performed using a combination of MATLAB Simulink tool and Simscape Multibody environment. The last one is very acceptable for block diagram modelling because it allows a block view of all sensors, bodies, system elements, joints and constraints using block components from the Simscape ${ }^{\mathrm{TM}}$ family. It also provides the possibility of $3 \mathrm{D}$ displaying the animation of the dynamics of the observed robot system. The control algorithm is derived using a PID controller on one robot leg (in each joint). The controller's parameters are adjusted on one leg of the robot, and after that, this is used to adjust the control on the other legs in this way. In joints 1 and 2 PID algorithm is based on position control [8]while in joint 3 is based on force control [8].

Below is a brief description of how this work is organized by its units. After this introduction, the next section briefly addresses some earlier research by the authors which this research is an extension of, while Section 3 describes the authors' motivation for conducting this research. Section 4 describes the hexapod robot product architecture, while Section 5 describes kinematic analysis with mathematical model of the hexapod robot. Section 6 presents control algorithm used and the next section presents conducted simulation, while Section 8 presents the obtained results with discussion. As the final section of the paper, Section 9 provides a conclusion for the research presented, as well as the possible directions of future research.

\section{LITERATURE REVIEW}

In this chapter, the authors give a brief overview of works in the field of creation, modelling and control of hexapod robots. We want to point out the work of authors Stoian and Vlado [9], who showed so-called two-tier (multi-hierarchical) governance structure. Namely, the control algorithm that is used in case of insufficient walking is presented in such way that it analyses the working space and gives commands to the robot joints. Its goal is to minimize robot movements in such way that the position is secured in an acceptable proximity to the desired position. There are also papers with an adaptive approach of robot motion control, like a paper of Quyang et al. [10]. The development of algorithms is based on the observed knowledge from nature, where a three-dimensional two-layer network of an artificial central sample generator (CPG) was created to generate the robot trajectory. The first layer of the generator generates several basic patterns of 
movement, while the second controls the behavior of the robot's legs, in such way that it adapts to changes in the environment in a particular pattern of movement. A motion simulation was performed, as well as an experiment. Some of the papers also show certain applications of robots for particular purposes, such as work of Gökrem and Can [11], which gives a comparison of two regulators PID and Fuzzy Logic, and would be applied to greenhouses, and for operations of searching of missing persons. and rescuing. The paper presents the structure of robots and based on that cognition, control algorithms are developed. There also can be found a presentation of some ready-made solutions such as the development of hexapod robots type RHex, by Saranli et al. [12] with six degrees of freedom of movement, where a robot simulation and an experiment in walking, running and turning a robot are shown. Like in this paper, there are many papers that conduct their simulation using the Simscape tool. One of such papers is the one of author Urrea et. al. [13] showing the application of MATLAB simulink tools, Simscape Multibody plugin and VRML language. A three-dimensional model of the robot is shown in the VRML language, while a PID controller is used for control to obtain a predetermined robot displacement. The work of Deng et al. [14] is interesting because it gives an overview of robot movement in rolling gait mode and trajectory planning. The idea is to constantly change the gait according to the different environment in which the robot moves, as well as the state of its stability. The CPG network is also used in the work of the author Campos [15], where the movement of hexapod robots on different types of terrain is shown, and the control is solved multi-hierarchically for three levels using a central sample generator. The work of the author Tedeschi and Carbone [16] provides an overview of robot movement planning with the aim of improving stability speed, navigation autonomy and energy efficiency. In addition, a classification of hexapod robots with their description is given.

\section{MOTIVATION}

With this paper, the authors wanted to present the beginning of their research on the internal project „KO006-2020/1 - Establishment and equipping of laboratory for the course „Systems and Control Algorithms in Robotics" and "Mobile Robotics” at the Polytechnic Graduate Professional Study of Electrical engineering" supported by Zagreb University of Applied Sciences, Zagreb, Croatia., which is currently in active phase. It is planned that part of the developed and presented algorithms for the movement of hexapod robots will be used as part of laboratory exercises in these courses. Also, by researching this area, authors wanted to expand their knowledge in the field of mobile robotics, and they wanted to share it with other young scientists who can also use it, as they are just like them at the beginning of their research.

\section{HEXAPOD ROBOT PRODUCT ARCHITECTURE}

When creating the architecture of the hexapod robot, the authors were guided by already known findings from published papers. Here we primarily mean studies of the movement of animals in nature and their natural forms [17]-[18]. As it is well known, many arthropods (such as insects and crustaceans) have six legs for the purpose of maintaining static stability and dynamic stability, and a certain robustness of their architecture during their movement. It is interesting that their body is made as segmented, which is covered with a connected outer skeleton. As one of the examples of hexapod motion in nature, we can give the example of a cockroach and the robot architecture derived from it, shown in Figure 1.
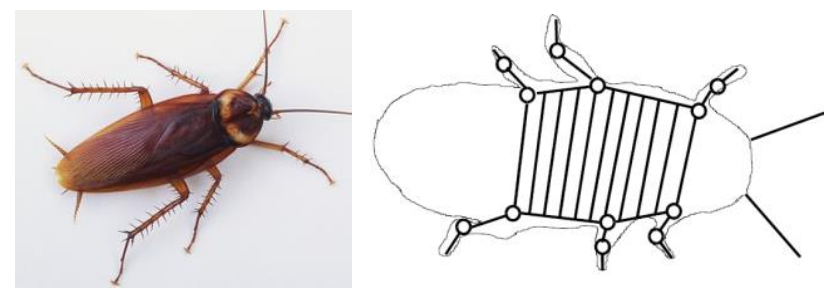

Figure 1. Example of anatomy of an insect from nature (cockroach) and based on that derived architecture of a hexapd robot shown by a simplified kinematic scheme 
A typical example of a robot developed on the abovementioned knowledge of insect anatomy and their movement is the hexapod robot [2]. What has been developed in them are already known modes of movement, one of which will be a tripod gait that will be considered during the control of this robot. The biggest problem is degrees of freedom of movement on the legs [4], and we can say that it is quite a complex problem to solve the control, ie to create a control algorithm. In hexapod robot architecture, we can find two main subgroups in the literature, namely rectangular and hexagonal robot architecture [17]. Both architectures are inspired by the appearance of insects, where the difference between them is in the symmetry of the position of all 6 legs along the body. In the rectangular design, this is done symmetrically, while in the hexagonal design, the legs are placed asymmetrically along the robot body. It is important to say that in the literature there is another division according to the type of legs of hexapod robots [19], where this type of robot is classified in the group of robots with bio-inspired legs in which are mammals, arachnids and reptiles.

In our work, we used the simplified architecture of the hexapod robot, which is shown in Figure 2 with all the legs, their ankles, and joints. It should be noted that each robot joint has a servo motor, which is controlled. Each leg is numbered and all the considered coordinate systems are placed on the diagram. According to the given scheme, the performed kinematic analysis of the robot will be presented in the next chapter of this paper.

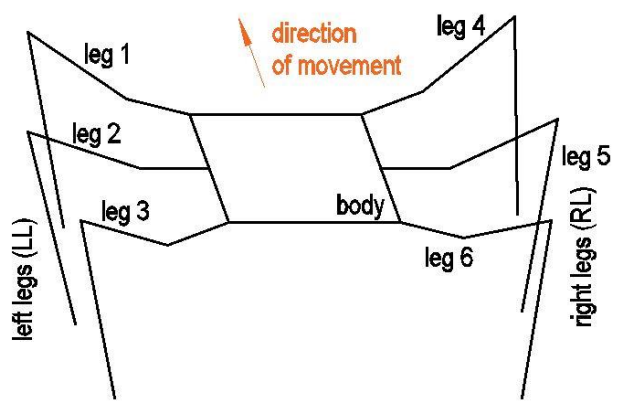

Figure 2. Representation of the considered hexapod robot, shown by a simplified kinematic scheme

\section{KINEMATIC AND DYNAMIC ANALYSIS WITH MATHEMATICAL MODEL}

In the previous chapter, we briefly described the architecture of the hexapod robot. Also, we showed its simplified configuration shown in the kinematic scheme in Figure 2. That is important to be to explained before conducting the kinematic analysis for the reason that we can predict all the effects of individual robot motion performance. Considering the symmetrical rectangular structure of the legs of the hexapod robot, it can be seen that we have 3 legs which are located on both sides, where each leg has 3 degrees of freedom of movement (3 DOF). In order to be able to perform an inverse kinematic model of a robot on this basis, we need to define coordinate systems for legs and body in the diagram, which later will be used to create all the different phases during the tripod gait movement of the robot. Coordinate systems were selected as follows (Figure 3):

$R_{0}\left(\mathrm{O}_{0}, \mathrm{X}_{0}, \mathrm{Y}_{0}, \mathrm{Z}_{0}\right)$ - global coordinate system

$R_{b}\left(\mathrm{O}_{\mathrm{b}}, \mathrm{X}_{\mathrm{b}}, \mathrm{Y}_{\mathrm{b}}, \mathrm{Z}_{\mathrm{b}}\right)$ - coordinate system of robot body

$R_{l i}\left(\mathrm{O}_{\mathrm{li}}, \mathrm{X}_{\mathrm{li}}, \mathrm{Y}_{\mathrm{li}}, \mathrm{Z}_{\mathrm{li}}\right)$ - coordinate system of robotic leg $(i=1,2, \ldots 6$ - number of robot leg $)$

Figure 3 already shows and observes the robot leg (leg no. 5, marked in purple, is selected here). It was created in such way that it has three degrees of freedom of movement, because it has already been shown that the minimum number of joints of a robot's leg is needed to ensure its mobility and movement in different types of gait. Thanks to the symmetrical configuration of the robot, it is enough to perform the analysis on only one leg of the robot. 


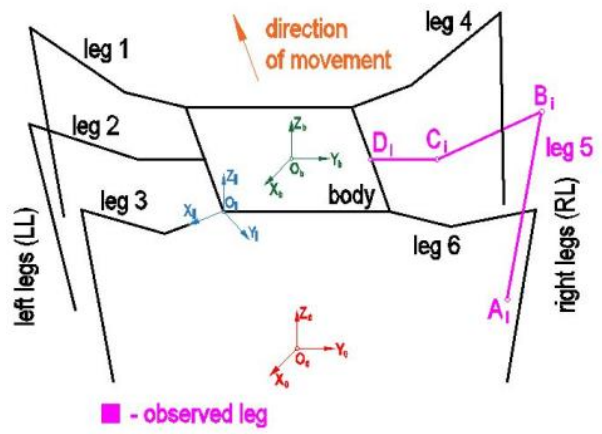

Figure 3. Simplified kinematic scheme of robots with defined oordinate systems

Individual parameters on each joint and variables on joints ( 1 - root joint, 2- hip joint and 3 - knee joint) are defined, and the equation of motion for the leg is obtained in next chapter. On each link of the presented open kinematic chain, right-wing orthonormal coordinate systems are systematically joined.

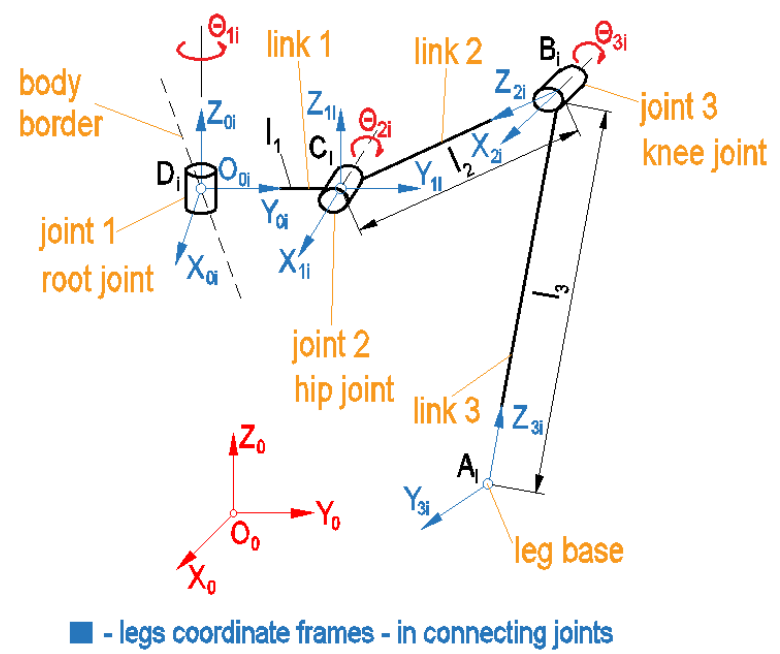

Figure 4. Simplified kinematic scheme of the observed leg of robot no. 5 with defined coordinate systems shown

When deriving the equations of motion of the hexapod robot, we use Denavit-Hartenberg's algorithm [7], which we will apply to the shown observation leg of the robot in Figure 4. In most literature [5]-[6] we will find the names denoting the marked points in the diagram in Figure 4. Thus joint 1 is called "thorax", link 1 is called "coxa", link 2 is called "femur", and link 3 is called "tibia". Mentioned algorithm is based on the systematic association of right-handed orthonormal coordinate systems to each open kinematic chain member. Since we have a more complex kinematic structure (3 DOF) here, the mathematical model of direct kinematics [7] is solved by relative or homogeneous coordinate transformations e.g. Denavit - Hartenberg parameters for the observed robot leg with 3 DOF have already been derived in the paper by the author Mănoiu-Olar [20], we present them here in tabular form (Table 1).

Table 1. Denavit - Hartenberg parameters for the observed robot leg (3 DOF)

\begin{tabular}{|c|c|c|c|c|}
\hline Link & $a_{i}$ & $\alpha_{i}$ & $d_{i}$ & $\Theta_{i}$ \\
\hline 1 & $1_{1}$ & $90^{\circ}$ & $d_{1}$ & $\Theta_{1}$ \\
\hline 2 & $1_{2}$ & $0^{\circ}$ & 0 & $\Theta_{2}$ \\
\hline 3 & $1_{3}$ & $180^{\circ}$ & 0 & $\Theta_{3}$ \\
\hline
\end{tabular}

A matrix of homogeneous transformation ${ }^{i-1} A_{i}$ can also be found in $[19,20]$, which connects the link $i-1$ with the link $i$. Based on it, a total transformation is performed later by multiplying the matrices ${ }^{0} A_{1},{ }^{1} A_{2}$ i ${ }^{2} A_{3}$ in order to obtain the resultant matrix representing the relationship 
between the global coordinate system and the coordinate system at the base of the robot leg ${ }^{0} A_{3}$. The solution of the mentioned system gives the equations of the direct kinematics of the tool, ie the base of the robot leg (tool configuration space) (5.1) - (5.3):

$p_{x}=\cos \theta_{1}\left(l_{1}+l_{2} \cdot \cos \theta_{2}+l_{3} \cdot \cos \left(\theta_{2}-\theta_{3}\right)\right)$

$p_{y}=\sin \theta_{1}\left(l_{1}+l_{2} \cdot \cos \theta_{2}+l_{3} \cdot \cos \left(\theta_{2}-\theta_{3}\right)\right)$

$\left.p_{z}=d_{1}+l_{2} \cdot \sin \theta_{2}+l_{3} \cdot \sin \left(\theta_{2}-\theta_{3}\right)\right)$

The next step in kinematic analysis is to determine the angles in a particular joint depending on the coordinates of that same joint in the coordinate system (joint space). Abovementioned variables will enable us when controlling the robot, and as a starting point we will use the equations of direct kinematics already defined. This problem has already been solved in the available literature [21], so here we list the obtained formulas (5.4) - (5.6):

$\Theta_{1}=\operatorname{artg}\left(\frac{y_{1}}{x_{1}}\right)$

$\Theta_{2}=\operatorname{arcos}\left(\frac{l_{2}^{2}+x_{3}^{2}+y_{3}^{2}-l_{3}^{2}}{2 \cdot l_{2} \cdot \sqrt{x_{3}^{2}+y_{3}^{2}}}\right)+\operatorname{artg}\left(\frac{y_{3}}{x_{3}}\right)$

$\Theta_{3}=\Pi-\arccos \left(\frac{l_{2}^{2}+l_{3}^{2}-\left(x_{3}^{2}+y_{3}^{2}\right)}{2 \cdot l_{2} \cdot l_{3}}\right)$

where are:

$l_{1}, l_{2}, l_{3}[\mathrm{~m}]-$ lengths of links 1,2 and 3 ,

$\theta_{1}\left[{ }^{\circ}\right]$ - angle closed by the robot body with link 1 ,

$\theta_{2}\left[{ }^{\circ}\right]$ - angle closed by link 1 with link 2 ,

$\theta_{3}\left[{ }^{\circ}\right]$ - angle closed by link 2 with link 3 .

In dynamic analysis, the purpose is to obtain the dynamic equations of motion of robot legs. For this purpose, it is necessary to consider all the necessary mass and geometry of the legs (length and thickness of the legs), and the geometry of the servo motor in order to obtain the most realistic dynamic model. The parameters used will calculate all the required volumes and the axial and centrifugal dynamic moments of inertia of the links and servo motors required to perform this analysis. Regarding this is an analysis of a multi-ankle leg, a much more applied model was chosen for this purpose, and that is the Lagrange-Euler model, which is needed due to the possibility of controlling the force of touch by a link 3 of the robotic leg. The model is based on the application of generalized coordinates and a combination of the following equations [8]: Lagrange equations of the second kind with Lagrange function $L(q, \dot{q})$, kinetic energy of the manipulator $T$ (includes the effects of translational and rotational motions), and potential energies $U$. Similar derived dynamic model for a robotic leg with 3 DOF-can already be found in the literature $[13,22]$. Due to the fact that all actuators (servo motors) in the joints are of the same mass, it is marked for all 3 servo motors with the same symbol $m_{S M}$. It should be emphasized that the masses of cables, associated electronics and other connecting elements were not considered in this analysis. The vector equation representing the dynamic model of the robotic arm [8] is written in the form:

$D(q) \ddot{q}+c(q, \ddot{q})+h(q)+b(\dot{q})=M_{t}$

where are:

$\boldsymbol{D}(\boldsymbol{q})$ - manipulator inertia tensor - symmetric matrix of dimensions $\mathrm{n} \times \mathrm{n}$,

$\boldsymbol{c}(\boldsymbol{q}, \ddot{\boldsymbol{q}})$ - connection vector of the $\mathrm{i}-$ th joint product of joint velocities and connection matrix of velocities $\mathrm{C}$ - matrix of dimensions $\mathrm{n} \times \mathrm{n}$ - represents centrifugal and Coriolis forces,

$\boldsymbol{h}(\boldsymbol{q})$ - vector of gravitational action - vector of dimensions $\mathrm{n} \times 1$ - describes the influence of gravity on the manipulator

$\boldsymbol{b}(\dot{\boldsymbol{q}})$ - represents the friction that opposes the movement of the robotic arm. 
$\boldsymbol{q}$ - vector of joint variables.

Below are the equations of actuator moments (5.8) - (5.10) obtained on the basis of the described approach:

$M_{t 1}=\ddot{\theta}_{1} \cdot\left(I_{m 1}+I_{l 1}+m_{S M} \cdot\left(l_{1}^{2}+r_{S M}\right)+m_{2} \cdot\left(r_{1}^{2}+r_{2}^{2}\right)\right)$

$M_{t 2}=\ddot{\theta}_{2} \cdot\left(I_{m 2}+I_{l 2}+m_{S M} \cdot l_{2}^{2}+m_{3} \cdot r_{3}^{2}\right)-g \cdot\left(l_{3} \cdot \cos \left(\theta_{2}+\theta_{3}\right) \cdot\left(3 m_{S M}+m_{1}+\frac{3 m_{2}}{2}\right)+\right.$

$\left.l_{2} \cdot \cos \theta_{2}\left(2 m_{S M}+m_{1}+\frac{m_{2}}{2}\right)\right)$

$M_{t 3}=\ddot{\theta_{3}} \cdot\left(I_{m 3}+I_{l 3}\right)-g \cdot l_{3} \cdot \cos \left(\theta_{2}+\theta_{3}\right) \cdot\left(3 m_{S M}+m_{1}+\frac{3 m_{2}}{2}\right)$

where are:

$M_{t 1}, M_{t 2}, M_{t 3}[\mathrm{Nm}]$ - actuator moment (servo motors) in joints 1, 2 and 3,

$I_{m 1}, I_{m 2}, I_{m 3}\left[\mathrm{~kg} \cdot \mathrm{m}^{2}\right]$ - dynamic moment of inertia of servo motor in joints 1,2 and 3 ,

$I_{l 1}, I_{l 2}, I_{l 3}\left[\mathrm{~kg} \cdot \mathrm{m}^{2}\right]$ - dynamic moment of inertia of robotic leg joints 1, 2 and 3,

$m_{S M}[\mathrm{~kg}]$ - mass of servo motor in joints 1, 2 and 3,

$m_{1}, m_{2}, m_{3}[\mathrm{~kg}]-$ mass of linksof the robot leg 1,2 and 3 ,

$r_{S M}[\mathrm{~m}]$ - radius of rotation of servo motor in joints 1,2 and 3 ,

$r_{1}, r_{2}, r_{3}[\mathrm{~m}]$ - radius of rotation of the center of mass in the links of the robot leg 1,2 and 3 ,

$\ddot{\theta_{1}}, \ddot{\theta_{2}}, \ddot{\theta_{3}}\left[\mathrm{~m} / \mathrm{s}^{2}\right]$ - angular accelerations in joints 1,2 and 3 .

The generalized contact force acting on the robotic manipulator [8] is calculated according to equation (5.11):

$\boldsymbol{F}=\boldsymbol{M}_{\boldsymbol{t}}-\boldsymbol{b}(\dot{\boldsymbol{q}})$

\section{DESCRIPTION OF USED ALGORITHM FOR HEXAPOD ROBOT CONTROL}

A PID controller has been selected to control the robot's ankle joint, i.e. the servo motor built into it. It is primarily chosen due to its derivational characteristic, thus improving the stability of the regulatory system. It is possible to increase the gain constant $K$, and at the same time reduce the integration constant $T_{i}$, which contributes to increasing the ability to more accurately monitor the reference quantity [23]. Today, this type of regulator is still the most common type of industrial regulators. The reason for this lies in the experience of the staff involved in their setup and commissioning, but also in their simplicity and comprehensibility of the control algorithm.

When developing a control algorithm, the simplest example of robot's usage locomotion on flat terrain is taken here.

The control signal of the PID controller can be written with the following expression [24]

$u(t)=K\left[e(t)+\frac{1}{T_{i}} \int_{0}^{t} e(\tau) d \tau+T_{d} \frac{d e(t)}{d t}\right]$

or in the another form

$u(t)=K e(t)+K_{i} \int_{0}^{t} e(\tau) d \tau+K_{d} \frac{d e(t)}{d t}$

where are:

$K$ - proportional gain constant of the regulator,

$K_{i}=K / T_{i}$ - gain constant (reset) of the integration part of the regulator,

$K_{d}=K \cdot T_{d}$ - gain constant of the derivation part of the regulator.

Usually, it takes a long time to set the parameters of the PID controller. On the one hand, it makes it easier for us to train the staff to do it (manual adjustment). On the other hand, the procedures for this purpose have already been developed. In this paper, the setting option in program MATLAB Simulink was used using the PID tuner window, correcting the values using the slider 
for response time and transient phenomena options.

The control structure for two joints 1 and 2 of one robot leg shown in Figure 5, where we can observe that there are two parts of the system: the part with the regulator and the part of the system that concerns the robot. The control of these two joints was performed by position (joint rotation angle). The mathematical model of robots and the problem of Inverse kinematics has already been described in more detail in the previous chapter of this article.

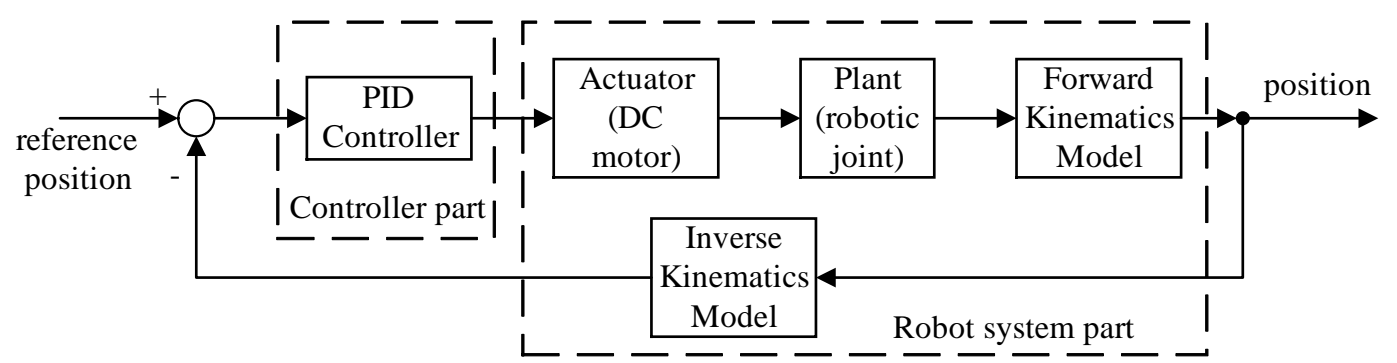

Figure 5. Display of the concept of control system for robotic joints 1 and 2

When used the contact force control on which the control of link 3 is based (because it has contact with the environment (ground)), it is necessary to install a contact force sensor that compares the measured force with the given once. In this case, the presentation of the management concept is shown in Figure 6.

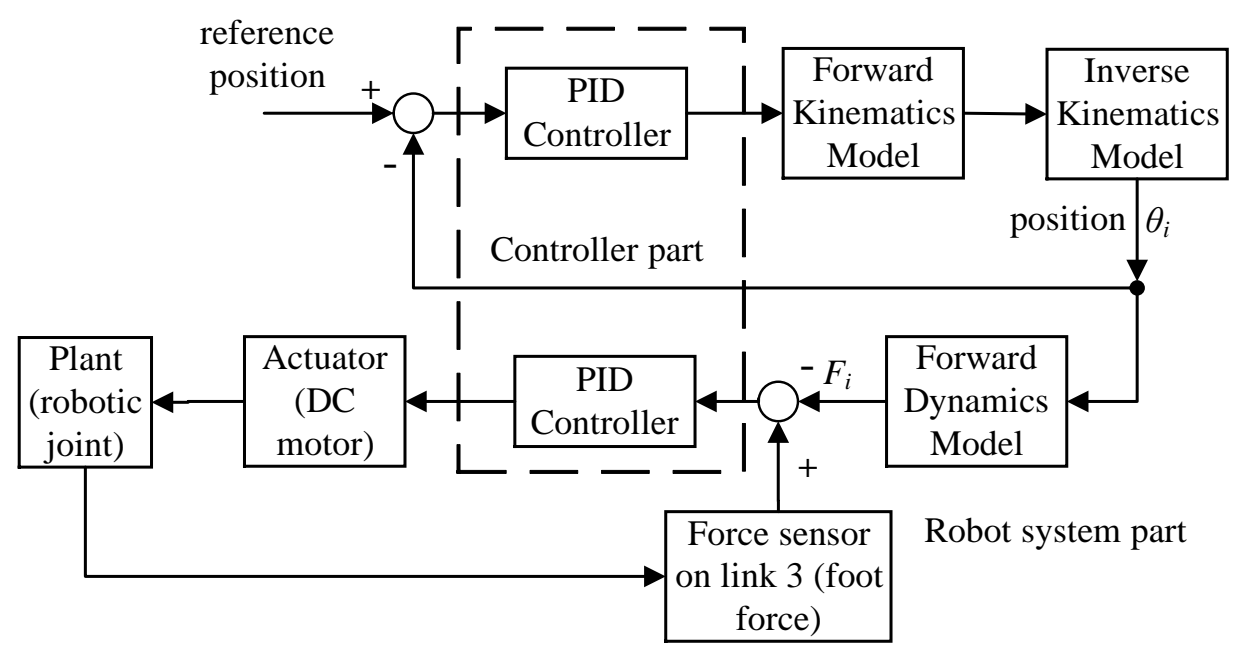

Figure 6. Display of the concept of control system for robotic joint 3

\section{SIMULATION}

Before creating the block model, it is necessary to calculate the parameters we need to create that model. For this purpose, Table 2 shows the values of the parameters that we used when calculating the dynamic moments of inertia [8] of the robot body and legs, as well as their masses (Note: for the dimensions of the robot body and legs, the overall dimensions were taken). When calculating the dynamic moments of inertia, we assumed that due to the symmetrical structure of the components the center of mass coincides with the center of gravity of the body. It is also assumed that the material of each component has homogeneous properties, i.e. that the material behaves the same in all directions of the component.

A combination of MATLAB Simulink and Simscape Multibody was used to create the simulation model. The use of the Simscape Multibody environment was very helpful because the hexapod robot has 6 legs, and each of them has 3 degrees of freedom of movement. This is the model itself, and it makes the behavior simulation quite complex. This environment has the advantage that it already created blocks for a certain type of joints, as well as the ankles of the legs. Each block already has the ability to individually adjust its parameters (geometry, mass, center of mass and moments of inertia). 
Table 2. Parameters needed to calculate the masses and dynamic moments of inertia of individual robot components

\begin{tabular}{|c|c|}
\hline \multicolumn{2}{|c|}{ Robot geometry } \\
\hline Dimensions of robot body $(L \times W \times H)[\mathrm{mm}]$ & $150 \times 70 \times 4$ \\
\hline Dimensions of link $1\left(l_{1} \times w_{1} \times h_{1}\right)[\mathrm{mm}]$ & $36 \times 13 \times 8$ \\
\hline Dimensions of link 2 $\left(l_{2} \times w_{2} \times h_{2}\right)[\mathrm{mm}]$ & $52 \times 10 \times 3$ \\
\hline Dimensions of link 3 $\left(l_{3} \times w_{3} \times h_{3}\right)[\mathrm{mm}]$ & $75 \times 15 \times 3$ \\
\hline Dimensions of servo motor $\left(\Phi D_{S M} \times l_{S M}\right)[\mathrm{mm}]$ & $\Phi 12 \times 28$ \\
\hline \multicolumn{2}{|c|}{ Material for robot body and legs and their density } \\
\hline duralumin & $\rho=2500 \mathrm{~kg} / \mathrm{m}^{3}$ \\
\hline
\end{tabular}

The PID block in the MATLAB Simulink tool was used for control in block model in MATLAB SImulink. It has already been said that the setting option within the MATLAB Simulink using the PID tuner window was used to set the parameters of the PID controller. When creating a motion trajectory, a rectilinear motion was used as a function of the direction given to the function in the block. When planning the trajectory of the movement of hexapod robots, the movement of the robot's leg in its stance (supporting) phase and swing (suspending) phase, and the transfer phase should be considered throughout the moving cycle.

Each leg has three joints, each joint contains a servo motor. In the starting position, angle in the joint $A$ is set to value $0^{\circ}$, in the joint $B$ to $45^{\circ}$ and in joint $C$ to value $30^{\circ}$. During robot moving, it is first planned to move legs 1, 3 and 5 (marked in Figure 1). After these legs touch the ground, the same movement is achieved by legs 2, 4 and 6 with the same amplitude and frequency of movement. In the presented simulation, the robot walk along a straight path of a certain distance was performed, and therefore the robot movement analysis was performed.

\section{DISCUSSION ON OBTAINED RESULTS}

Figures 7 and 8 show the obtained responses of the moments in the joints 2 and 3 of the observed robotic leg after the performed simulation. It should be noted that the changes in the angles in the joints are set to vary in the range from $-45^{\circ}$ to $45^{\circ}$ for the joint 2 , and in the range from $-30^{\circ}$ to $30^{\circ}$ for the joint 3 . What should be mentioned is that when modeling the kinematic and the dynamic model of the robot, individual phases during the rotation of the legs were not considered. Namely, the movement of the robot's leg in its stance (supporting) phase, swing (suspending) phase, and the transfer phase should be considered throughout the moving cycle. Some models have already been derived on this topic, as shown in [25].

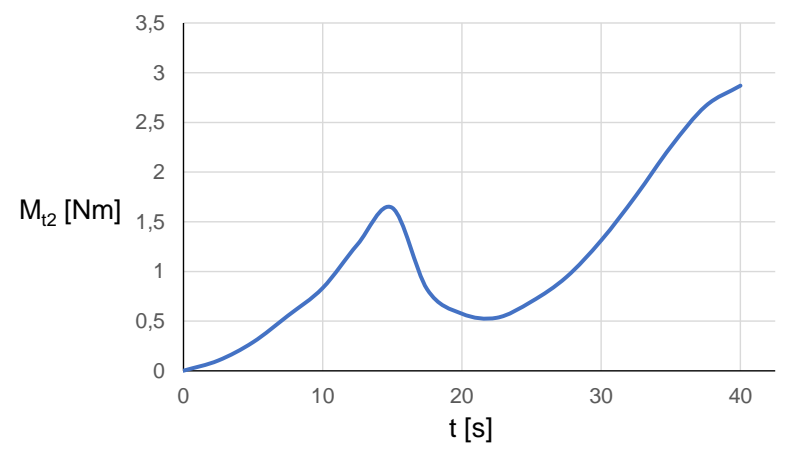

Figure 7. Display of the change of moment in the joint 2 of observed robotic leg 


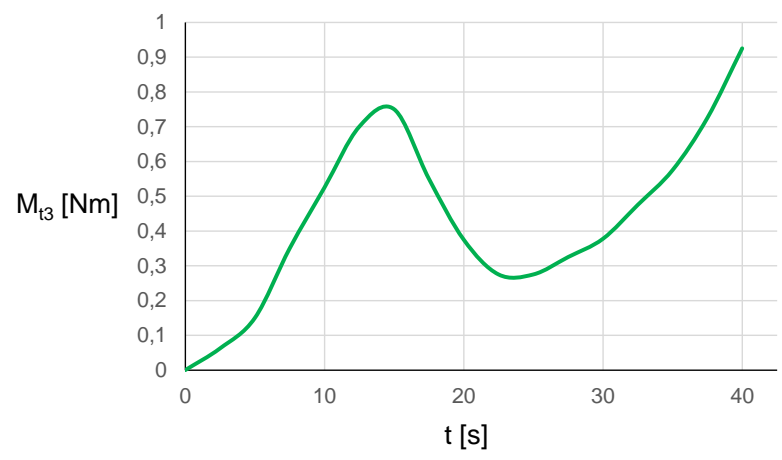

Figure 8. Display of the change of moment in the joint 3 of observed robotic leg

\section{CONCLUSION AND SOME POSSIBLE WAYS OF FUTURE RESEARCH}

In this paper, a development of mathematical model and the control algorithm of a hexapod robot during tripod gait locomotion is described. The kinematic analysis was done to obtain a mathematical model of the robot. It was done using the Denavit-Hartenberg (D-H) method and homogenous transformations to establish the joint coordinate system of a hexapod robot for its one leg. Solving the problem of inverse kinematics gives the values of the angles of rotation for each of the joints, for a given point in space at the observed robot leg. During hexapod robot trajectory planning, the movement of the robot's leg in its stance (supporting) phase and swing (suspending) phase, and the transfer phase should be considered throughout the moving cycle. Simulation is performed using combination of computer tool MATLAB Simulink and Simscape Multibody environment. The intention is to take advantage of that, so that could be used in teaching laboratory exercises in one of the courses at the graduate study on Zagreb University of Applied Sciences. The mathematical model presented is derived according to a model based on rectangular hexapod design configuration, using PID controllers in each leg joint. During the conduct of the research it has been proven that a major drawback is use of a PID controller which is quite difficult to set their controller parameters. When developing a control algorithm, the simplest example of robot's usage locomotion on flat terrain is taken here.

The development and application of another regulator based on adaptive control, as well as, its application for these robots on uneven terrains, has been presented as several directions for future research. The intention is to develop algorithms for other standard types of gait hexapod robots, and compare them with each other. It would also be good to make a prototype of the described robot, and conduct experimental results in order to confirm the simulation results.

\section{ACKNOWLEDGMENTS}

The research presented in this paper is part of internal project „KO006-2020/1 - Establishment and equipping of laboratory for the course „Systems and Control Algorithms in Robotics" and „Mobile Robotics" at the Graduate Study of Electrical Engineering at Zagreb University of Applied Sciences, Zagreb, Croatia.

\section{REFERENCES}

[1] M. Shahriari. Design, Implementation and Control of a Hexapod Robot using Reinforcement Learning Approach”, M.Sc. Thesis, Kish Island, Iran, 2013.

[2] S. Mănoiu-Olaru and M. Niţulescu. Matlab Simulator for Gravitational Stability Analysis of a Hexapod Robot. The Romanian Review Precision Mechanics, Optics \& Mechatronics, No. 39, 2011.

[3] J. A. Tenreiro Machado and M. F. Silva. An Overview of Legged Robots. MME 2006 - International Symposium on Mathematical Methods in Engineering, Ankara, Turkey, 2006.

[4] G. Carbone and M. Ceccarelli. Legged Robotic Systems”, In: Cutting Edge Robotics ARS Scientific Book, Wien, pp. 553-576, 2005. 
[5] R. Campos, V. Matos, Cristina Santos. Hexapod locomotion: A nonlinear dynamical systems approach. IECON 2010 - 36th Annual Conference on IEEE Industrial Electronics Society

[6] X. Duan, W. Chen, S. Yu and J. Liu. Tripod gaits Planning and Kinematics Analysis of a Hexapod Robot. 2009 IEEE International Conference on Control and Automation, Chistchurch, New Zealand, pp. 1850-1855, 2009.

[7] R.P. Paul. Robot Manipulators: Mathematics, Programming and Control, The Computer Control of Robot Manipulators. The MIT Press, Cambridge Massachusetts and London, England, 1981.

[8] Z. Kovačić, S. Bogdan, V. Krajči. Osnove robotike. Graphis, Zagreb, Croatia, 2002.

[9] V. Stoian, I. C. Vladu. A Control Algorithm for Hexapod Mobile Robot Gait in Fault Conditions, 20th International Conference on System Theory, Control and Computing (ICSTCC), Sinaia, Romania, pp. 349-354, 2016.

[10]W. Ouyang, H. Chi, J. Pang, W. Liang and Q. Ren. Adaptive Locomotion Control of a Hexapod Robot via Bio-Inspired Learning, Frontiers in Neurorobotics, open acess, doi: 10.3389/fnbot.2021.627157

[11]L. Gökrem and M. Serhat Can. Hexapod Robot Design and Performance Comparison of Fuzzy and PID Control Methods, Balkan Journal of Electrical and Computer Engineering, Vol. 8, No. 1, pp. 8897, 2020.

[12]U. Saranli, M. Buehler and D. E. Koditschek. Design, Modeling and Preliminary Control of a Compliant Hexapod Robot, IEEE International Conference on Robotics and Automation, Vol. 3, ICRA 2000, pages 2589-2596, 2000.

[13]C. Urrea, L. Valenzuela and J. Kern. Design, Simulation, and Control of a Hexapod Robot in Simscape Multibody, InTech Open, open science, Applications from Engineering with MATLAB Concepts, chapter 5, pp. 125-137, 2016.

[14]H. Deng, G. Xin, G. Zhong and M. Mistry. Gait and trajectory rolling planning and control of hexapod robots for disaster rescue applications, Robotics and Autonomous Systems, 95, pp. 13-24, 2017.

[15]R. D. Costa Campos. Hexapod Locomotion: a Nonlinear Dynamical Systems Approach. MSc thesis, Universidade do Minho Escola de Engenharia, Portugal. 2009.

[16]F. Tedeschi and G. Carbone. Hexapod Walking Robot Locomotion, Motion and Operation Planning of Robotic Systems, Mechanisms and Machine Science, 29, pp. 439-468, 2015.

[17]X. Ding, Z. Wang, A. Rovetta and J.M. Zhu. Locomotion analysis of hexapod robot, in book: Climbing and Walking Robots, Behnam Miripour (Ed.), InTech, Vienna, Austria, pp. 291-310., 2010.

[18]S. Mănoiu-Olaru, M. Niţulescu. Stability Analysis Software Platform Dedicated for a Hexapod Robot, in book: Advances in Intelligent Control Systems and Computer Science, vol. 187, Loan Dumitrache (Ed.), Springer Nature Switzerland AG, pp. 143-156, 2019.

[19]R. Shirpurkar. Investigation of Effects of Changing Length Scales of Uniformly Structured Rough Terrain on Hexapedal Locomotion using Simulation, M.Sc. thesis, University of California, San Diego, USA, 2'19.

[20]S. Mănoiu-Olaru. Hexapod robot. Mathematical support for modeling and control. In: System Theory, Control, and Computing (ICSTCC), 2011 15th International Conference, Sinaia. IEEE; pp. 1-6., 2011.

[21]R.K. Barai, P. Saha, and A. Mandal. SMART-HexBot: Simulation, Modeling, Analysis and Research Tool for Hexapod Robot in Virtual Reality and Simulin. In: AIR '13 Proceedings of Conference on Advances in Robotics, Pune, India, 2013.

[22]M. Nitulescu, M. Ivanescu, V. D. Hai Nguyen and S. Manoiu - Olaru. Designing the Legs of a Hexapod Robot, In: 2016 20th International Conference on System Theory, Control and Computing (ICSTCC), Sinaia, Romania, pp. 119-124, 2016.

[23]Z. Vukić, Lj. Kuljača. Automatsko upravljanje - analiza linearnih sustava, Kigen, Zagreb, Hrvatska, 2005.

[24]K.J. Astrom, T. Hagglund. PID Controllers: Theory, Design, and Tuning, second edition, International Society for Measurement and Control, Research Triangle Park, NC, USA, 1995.

[25]E. H. Hasnaa, B. Mohammed. Planning tripod gait of an hexapod robot, In: 14th International MultiConference on Systems, Signals \& Devices (SSD), pp. 163-168, 2017. 